Proceedings

\title{
The Multifunctional Roles of Chitosan in the Formation of Flower-Shaped Palladium Nanoparticles
}

\author{
Thi Tuong Vy Phan ${ }^{1,2, *}$
}

Citation: Phan, T.T.V. The multifunctional roles of chitosan in the formation of flow-er-shaped palladium nanoparticles. Mater. Proc. 2021, 3, x.

https://doi.org/10.3390/xxxxx

Published: 22 February 2021

Publisher's Note: MDPI stays neutral with regard to jurisdictional claims in published maps and institutional affiliations.

Copyright: (c) 2020 by the authors. Submitted for possible open access publication under the terms and conditions of the Creative Commons Attribution (CC BY) license (http://creativecommons.org/licenses /by/4.0/).
1 Center for Advanced Chemistry, Institute of Research and Development, Duy Tan University, 03 Quang Trung, Hai Chau, Danang 550000, Vietnam

2 Faculty of Environmental and Chemical Engineering, Duy Tan University, 03 Quang Trung, Hai Chau, Danang 550000, Vietnam

* Correspondence: phanttuongvy4@duytan.edu.vn

† Presented at the 1st International Electronic Conference on Metallurgy and Metals, 22 February-07 March 2021; Available online: https://iec2m.sciforum.net/

\begin{abstract}
The chemical and physical routes are usually used to synthesis metal nanoparticles. However, the harmful effects on the environment and human health turn scientists into finding greener methods. We have developed the novel green method for the synthesis of the flower Pd nanoparticles based on the chitosan (CS) polymer. In this method, CS can work as a stabilizer, a shape-directing agent, and a size-controllable agent for the synthesis of these nanoparticles. This study provided pioneer evidence about the multifunctional roles of natural polymer in the preparation of metal nanoparticles. Deep and extensive studies should be conducted to explore the great benefits of natural polymers in the green synthesis of metal nanoparticles to explore the great benefits of natural polymers in the green synthesis of metal nanoparticles.
\end{abstract}

Keywords: chitosan; stabilizer; size-controllable; shape-controllable; green synthesis

\section{Introduction}

Palladium (Pd) nanoparticles are emerging as an outstanding metal material in the nanotechnology field. They have excellent physical and chemical properties such as high thermal and chemical stability, high photocatalytic activity, and low cost [1]. Pd nanomaterials have been applied in the wide ranges in hydrogen storage/sensing, organic coupling synthesis, hydrogen detection, fuel cells, and sensors [2]. Pd nanostructures acting as photocatalysts, prodrug activators, and antimicrobial agents have also been discovered. The biomedicine applications of Pd nanoparticles have been discovered recently [3,4]. To reduce the cost and the number of toxic materials of the chemical and physical method, the green methods have been focused to discover recently. Herein, we reported the simple and scalable method to synthesis Pd nanoparticles. CS, which is a natural polymer, has biodegradability, biocompatibility, nontoxicity, and antibacterial activity. CS is a good stabilizer for metal nanoparticles [5]. In this work, we experimented with the effect of CS amount on the Pd nanoparticles. Vitamin C as act the mild reducing agent in this method. Interestingly, we observed that CS not only worked as a stabilizer but also as a shapedirecting and size-control agent for the synthesis of FPNPs. The size of FPNPs ranges from 25 to $150 \mathrm{~nm}$. The resulted Pd nanoparticles have good biocompatibility, strong absorption in NIR range, and good photothermal conversion.

\section{Materials and Methods}

\subsection{Materials}


Palladium chloride (PdCl2), CS (50 to $190 \mathrm{kDa}$ and $75-85 \%$ degree of deacetylation), L-ascorbic acid, and polyvinyl alcohol (PVA, 89 to $98 \mathrm{kDa}$ ).

\subsection{One-spot synthesis of Pd nanoparticles}

The Pd nanoparticles were synthesized by the one-pot method. Firstly, a defined amount of CS and $50 \mathrm{mg}$ ascorbic acid were adding to the baker with $15 \mathrm{Ml}$ distilled water. Next, the various amount of CS was used to obtain different mass concentration (C \%) of CS. Then, $10 \mathrm{ml} \mathrm{HPdCl}{ }_{4} 0.01 \mathrm{M}$ was dropped to the CS solution. The nanoparticles solution was left for aging in $2 \mathrm{~h}$. Finally, the resulted nanoparticles were collected by centrifugation and washing three times with distilled water. The same procedure was used to synthesis Pd nanoparticles for control without adding CS.

\section{Result and Discussion}

\subsection{Chitosan as a stabilizer for the synthesis of Pd nanoparticles}

Two experiment conditions were set up to evaluate the effect of CS on the formation of Pd nanoparticles (CS reaction and no-CS reaction). The TEM images revealed that the no-CS reaction produced the big-size nanoparticles. Meanwhile, the CS reaction produced small size nanoparticles. The resulted solution was left for one day. To no-CS reaction, the aggregation was observed in the bottom of the tube; meanwhile, the CS reaction solution has no aggregation. The results again proved that CS is an excellent stabilizer for metal nanoparticles.
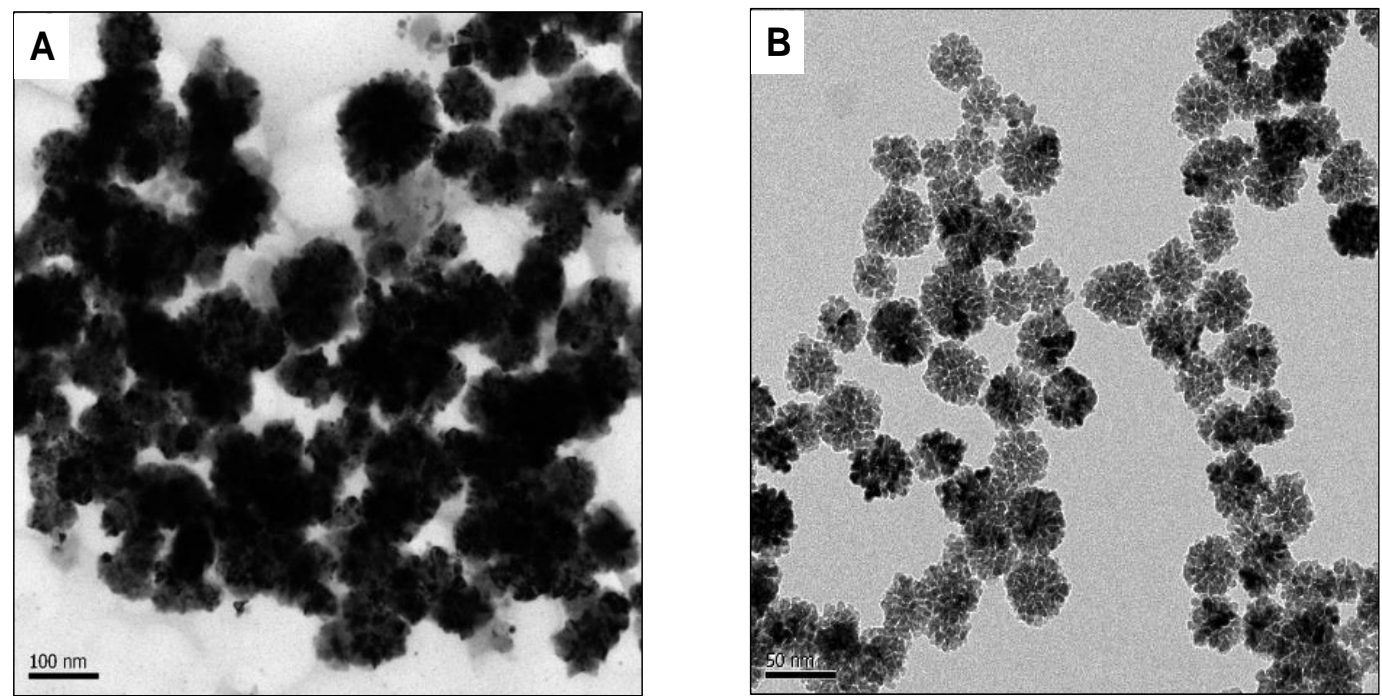

Figure 1. Morphologies of the synthesized Pd nanoparticles without CS (A) and with CS (B).

3.2. Chitosan as a size-control agent and shape-directing agent for the synthesis of flower Pd nanoparticles

The effect of CS on the formation of Pd nanoparticles was evaluated by setting six experimental conditions with different CS amounts. The TEM images were captured for morphology analysis. The nanoparticles have a flower shape with a range of sizes. The $\mathrm{Pd}$ nanoparticles, which were prepared with $0.05 \%(\mathrm{~m} / \mathrm{v}) \mathrm{CS}$, have the size of a nanoparticle of $153.7 \pm 59.7 \mathrm{~nm}$. The increasing the mass concentration of CS led to the decreasing in the size of the nanoparticles of resulted nanoparticles as shown in Figure 2. 


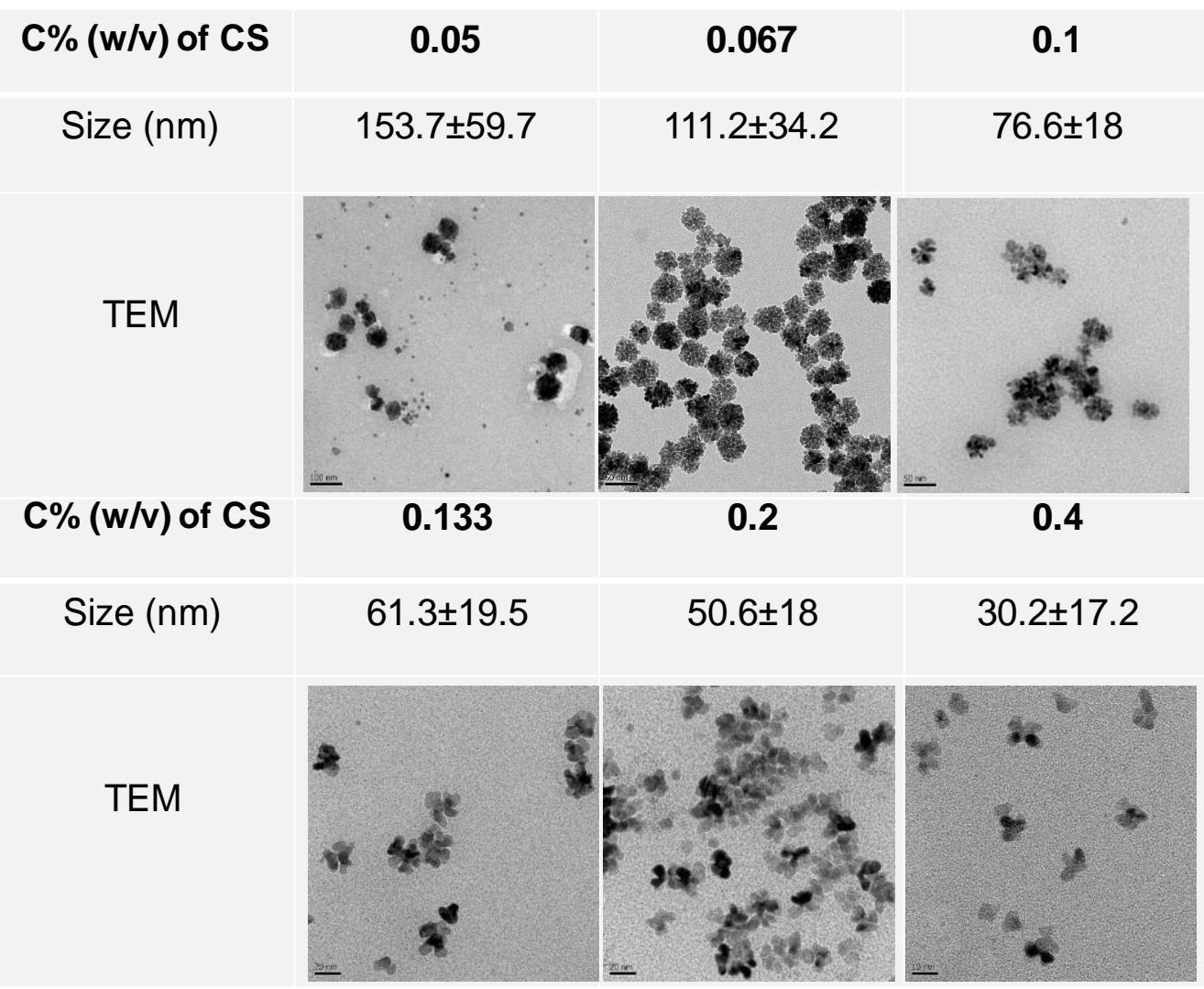

Figure 2. Morphologies and nanoparticle size of Pd nanoparticles synthesized with different C\% (m/v) of CS.

\section{Conclusion}

In conclusion, CS acted as multifunctional agents in the developed green synthesis method for the preparation of Pd nanoparticles. CS acted as a stabilizer, a size-control agent, and the shape-directing agent. The Pd nanoparticles can be controlled by adjusting the CS amount. This method is useful for preparing Pd nanoparticles with desired sizes that are suitable for different applications.

Funding: This research received no external funding.

Institutional Review Board Statement: Not applicable.

Informed Consent Statement: Not applicable.

Data Availability Statement: Data is contained within the article.

Acknowledgments: In this section, you can acknowledge any support given which is not covered by the author contribution or funding sections. This may include administrative and technical support, or donations in kind (e.g., materials used for experiments).

Conflicts of Interest: The authors declare no conflict of interest.

\section{References}

1. Chen A, Ostrom C. Palladium-Based Nanomaterials: Synthesis and Electrochemical Applications. Chemical Reviews. 2015 2015/11/11;115(21):11999-12044.

2. Chen Y-H, Hung H-H, Huang MH. Seed-Mediated Synthesis of Palladium Nanorods and Branched Nanocrystals and Their Use as Recyclable Suzuki Coupling Reaction Catalysts. Journal of the American Chemical Society. 2009 2009/07/01;131(25):91149121.

3. Kang S, Shin W, Kang K, et al. Revisiting of Pd Nanoparticles in Cancer Treatment: All-Round Excellence of Porous Pd Nanoplates in Gene-Thermo Combinational Therapy. ACS Applied Materials \& Interfaces. 2018 2018/04/25;10(16):13819-13828.

4. Lee J-H, Youn J-I, Kim Y-J, et al. Effect of Palladium Nanoparticles on Photocatalytic Characteristics of N doped Titania Catalyst. Journal of Materials Science \& Technology. 2015 2015/06/01/;31(6):664-669. 
5. Collado-González M, Montalbán MG, Peña-García J, et al. Chitosan as stabilizing agent for negatively charged nanoparticles. Carbohydrate Polymers. 2017 2017/04/01/;161:63-70. 